\title{
Contribution of the Advanced Research Laboratories at the Noguchi Memorial Institute for Medical Research (NMIMR) to SARS-CoV-2 Testing in Ghana
}

\author{
Darius N. K. Quansah ${ }^{1,2}$, Ivy A. Asante1, Evelyn Y. Bonney, ${ }^{1,2}$, Evangeline Obodai ${ }^{1}$, \\ Mildred A. Adusei-Poku',3, James Aboagye ${ }^{1,4}$, Susan Adu-Amankwah', Samuel Adjei6, \\ Richard Akuffo5, Helena Lamptey7, George B. Kyei'1,4, John K. Odoom1, \\ William K. Ampofo1,2, Joseph H. K. Bonney1,2*
}

\begin{abstract}
${ }^{1}$ Department of Virology, Noguchi Memorial Institute for Medical Research, University of Ghana, Legon, Accra, Ghana ${ }^{2}$ West African Centre for Cell Biology of Infectious Pathogens, College of Basic and Applied Sciences, University of Ghana, Legon, Accra, Ghana

${ }^{3}$ Department of Medical Microbiology, University of Ghana Medical School, Accra, Ghana

${ }^{4}$ Medical and Scientific Research Directorate, University of Ghana Medical Centre, Legon, Accra, Ghana

${ }^{5}$ Department of Epidemiology, Noguchi Memorial Institute for Medical Research, University of Ghana, Legon, Accra, Ghana

${ }^{6}$ Department of Animal Experimentation, Noguchi Memorial Institute for Medical Research, University of Ghana,

Legon, Accra, Ghana

${ }^{7}$ Department of Immunology, Noguchi Memorial Institute for Medical Research, University of Ghana, Legon, Accra, Ghana

Email: ^Kbonney@noguchi.ug.edu.gh
\end{abstract}

How to cite this paper: Quansah, D.N.K., Asante, I.A., Bonney, E.Y., Obodai, E., Adusei-Poku, M.A., Aboagye, J., Adu-Amankwah, S., Adjei, S., Akuffo, R., Lamptey, H., Kyei, G.B., Odoom, J.K., Ampofo, W.K. and Bonney, J.H.K. (2022) Contribution of the Advanced Research Laboratories at the Noguchi Memorial Institute for Medical Research (NMIMR) to SARS-CoV-2 Testing in Ghana. Health, 14, 125-136.

https://doi.org/10.4236/health.2022.141010

Received: October 18, 2021

Accepted: January 23, 2022

Published: January 26, 2022

\begin{abstract}
In March 2020, the first cases of SARS-CoV-2 were reported in Accra, Ghana. These initial cases were diagnosed at the Advanced Research Laboratories (ARL) of the Noguchi Memorial Institute for Medical Research (NMIMR), University of Ghana. The ARL which hitherto was used for routine clinical research in viral, bacteria and immunological studies has since been the facility of choice for testing for all suspected cases of COVID-19 submitted from across Ghana and beyond. The success of testing at the ARL hinged on the availability of several laboratory spaces furnished with state-of-the-art diagnostic equipment and working aids. During the "peak season" where overwhelming numbers of clinical specimens were received, the ARL processed and got results for close to four thousand samples daily. After general disinfection and re-bagging into smaller numbers, at the entrance of the ARL, the samples are taken to a central receiving laboratory, where they are received and entered in a database with accompanying case investigation forms. All
\end{abstract}


Copyright (C) 2022 by author(s) and Scientific Research Publishing Inc. This work is licensed under the Creative Commons Attribution International License (CC BY 4.0).

http://creativecommons.org/licenses/by/4.0/ samples that are successfully sorted and matched are sent to general laboratories for nucleic acid extraction and then referred to the Instrumentation laboratory for real time reverse-transcription polymerase chain reaction (RTPCR). When the RT-PCRs were completed, results were analysed and transmitted via email and/or local network to the data reporting office. The data managers then reported results to the investigators and the Ghana Health Service (GHS). Additionally, the ARL provided a next-generation Genome Sequencing platform in partnership with the West African Centre for Cell Biology of Infectious Pathogens at the University of Ghana, which was essential in reporting the genome data of the circulating variants of SARS-CoV-2 in Ghana. Conclusively, it is worth noting, that the NMIMR fulfilled its mandate of supporting the country with specialized diagnostics through the judicious use of the ARL for SARS-CoV-2 testing, from sample receipt to data reporting. The ARL facility and the research faculty have trained and continue to train budding laboratories on biosafety, biosecurity, best practices and testing protocols. It is obvious that the success story of SARS-CoV-2 testing in Ghana, cannot be complete without the mention of the ARL at NMIMR.

\section{Keywords}

Advanced Research Laboratories, Polymerase Chain Reaction, Severe Acute Respiratory Syndrome-Corona Virus 2, Corona Virus Disease 2019

\section{Introduction}

The COVID-19 pandemic which has claimed nearly four million lives and increased morbidity in the last year, continues to live with many communities [1] [2]. Despite the dip in the number of cases in many countries recently, an efficient testing regime is crucial to further halt transmission [3] [4]. On $12^{\text {th }}$ March 2020, Ghana recorded the first cases of severe acute respiratory syndrome-Corona Virus 2 (SARS-CoV-2), following a successful confirmatory testing at the Noguchi Memorial Institute for Medical Research (NMIMR) [5] [6] [7]. Days later, other cases were confirmed at the Kumasi Centre for Collaborative Research in Tropical Medicine (KCCR) [8]. Like many other countries, SARS-CoV-2 testing was initially done in academic research centres in Ghana because the national reference laboratories were inadequately equipped [1] [6] [9] [10]. The two academic institutions championed the early stages of SARS CoV-2 testing in Ghana, due to the available expertise and the advanced testing tools for coronaviruses and other emerging and re-emerging infectious pathogens.

Ghana has tested estimated one million and two hundred thousand samples, as at end of April 2021, since the first tests conducted in March 2020. Of this number, 90,700 were positive for SARS-CoV-2 and a near two-thirds of the tests were conducted at NMIMR, due to the availability of expanded resources at the 
ARL [GHS COVID UPDATES, APRIL 2021].

The NMIMR is a Biomedical Research Centre in the College of Health Sciences, at the University of Ghana, Legon. The Institute houses key regional and national reference laboratories and centres including the Regional Polio Reference Laboratory, the National Influenza Centre (NIC), the National HIV Genotyping laboratory and the World Health Organisation (WHO) National Tuberculosis Control program office. The Institute also serves the need for training Graduate biomedical scientists from universities in Ghana and the sub-region.

The ARL, which was commissioned in March 2019 was built and equipped with a Japanese Government Grant Aid, because of the long-standing friendship between the two countries. The building, which was subsequently furnished by the Government of Ghana, is one of the many benefits that the NMIMR has enjoyed from the people and government of Japan. The friendship between the two countries dates to 1920 when a renowned Japanese scientist, Dr. Hideyo Noguchi arrived in the Gold Coast to pursue his research on Yellow Fever virus. Dr Hideyo however died in the line of duty, from infection with the same yellow fever virus he was working on [11].

The ARL houses three main Departments of the Institute, Virology, Bacteriology and Immunology. All three departments have "General/All-purpose" laboratories, and special laboratories for specific laboratory activities including Media preparation, Virus Isolation and propagation, Cell culture, Washing and sterilization laboratories, a refrigerator $\left(2^{\circ} \mathrm{C}-8^{\circ} \mathrm{C}\right)$ room and a freezer $\left(-20^{\circ} \mathrm{C}\right)$ room and a designated room with biomedical refrigerators and freezers. Additionally, the ARL houses two Biosafety Level three (BSL-3) laboratories, one for bacteriology and the other for virology, and offices for research faculty, laboratory technicians and a common room big enough for an estimated 150 research assistants. The building also has a seminar room and an office suite for the Quality and Maintenance units of the Institute. The building is access-controlled with laboratory access granted to only authorized persons. The rooms at the ARL are well-spaced, and functionally organised in accordance with International Standard Laboratory Practices, which allows for a safe, regulated research at all levels.

The initiation of SARS-CoV-2 diagnosis by the National Influenza Centre (NIC) followed weeks of training to build capacity for research assistants and technicians. The NIC collaborates with the Centre for Disease Control (Africa and the USA), the World Health Organisation (WHO) and other developmental partners including US NAMRU-3 in surveillance of influenza and other respiratory infections in Ghana. The SARS-CoV-2 testing capacity building included training of key staff in Senegal on SARS-CoV-2 specimen handling, diagnosis and procurement of laboratory reagents and consumables in anticipation of likely events. The training and early testing for SARS-CoV-2 in Ghana was done with support from the Africa Centre for Disease Control (CDC) and the JACK MA Foundation, China providing laboratory reagents and other supplies [7]. 
Following the announcements of the confirmed cases, the government of Ghana instituted measures to carefully screen all passengers arriving in the country through air and land borders because the initial cases were imported. Passengers were quarantined mandatorily in selected hotels and other designated holding centres while arrangements were made to get COVID-19 tests done for them at the NMIMR. Trained health practitioners and laboratory personnel were dispatched daily to these holding facilities to collect respiratory specimen which were brought to NMIMR for testing. While this was being done, cases of community transmission which had been reported necessitated the need for contact tracing. Ghana as a country, adopted the enhanced community contact tracing (ECCT) module of investigation. The module required mass testing of primary and secondary contacts of positive cases. This soon resulted in overwhelming numbers of cases pending testing. The sample numbers increased rapidly by the days and soon all the laboratories of the ARL as well as the foyer of the cold rooms at the ARL were filled with large boxes containing samples yet to be tested. Piles of case investigation (CI) forms accompanied the samples submitted from nearly all 16 regions in Ghana. Information from these forms had to be transcribed manually into hardcover notebooks by staff. As tedious as this was, it was the only way the data was captured from the CI forms at the initial stages of testing. Later, the data was captured electronically, and staff spent days and nights capturing data from the $\mathrm{CI}$ forms into the database. The $\mathrm{CI}$ forms, which hitherto had flooded every desk in the offices were subsequently catalogued appropriately and shelved in giant spacious cabinets situated in the foyer of the ARL. Subsequently, an electronic data capturing tool (Surveillance Outbreak Response Management and Analysis System) was introduced to replace the CI forms accompanying the samples. This subsequently reduced drastically the load of paper CI forms that cluttered sections of the ARL.

\section{Workflow}

At the ARL, samples are first received from investigators or their representatives, mainly Disease Control Officers (DCO) at the main entrance. There is enough space at the forecourt of the ARL, where many vehicles (sometimes ambulances)which have been designated by the facilities for the purposes of transporting suspected COVID-19 samples, and in some instances having the inscription, "Fight Against COVID-19", park and await their turns to submit their clinical specimens. When it's your turn to be seen, you are first assisted by NMIMR staff on duty to pack out from the vehicles, cool boxes with samples for sample-adequacy inspection and cross linking and tallying case investigation (CI) forms with samples (Figure 1(a)). This initial sample receipt formality requires attention and therefore takes time which tends to frustrate some clients and the DCOs as they feel and complain of delays (Figure 1(b)). The sample receipt team disinfects containers or cool boxes, re-bag samples in groups of ten and proceed to record the number of samples being submitted for testing after physical inspection of the documents and the samples. There is a special desk at the 


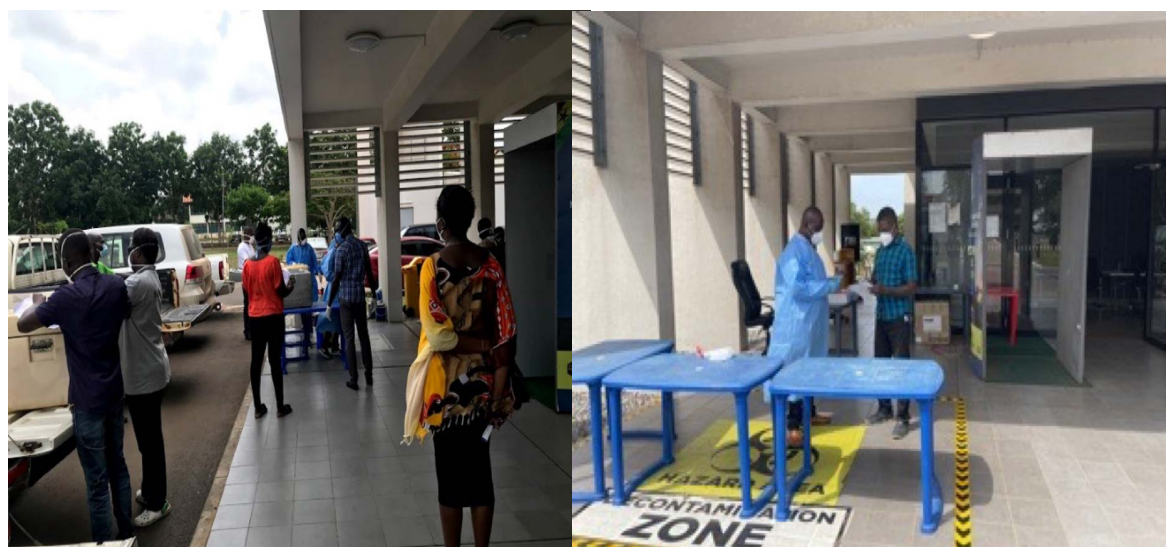

(a)

(b)
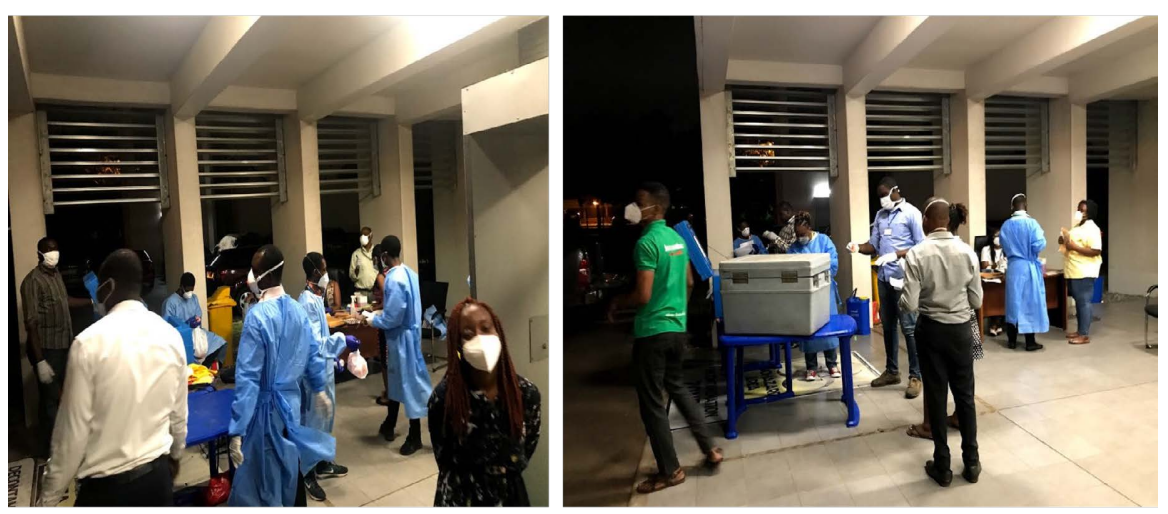

(c)
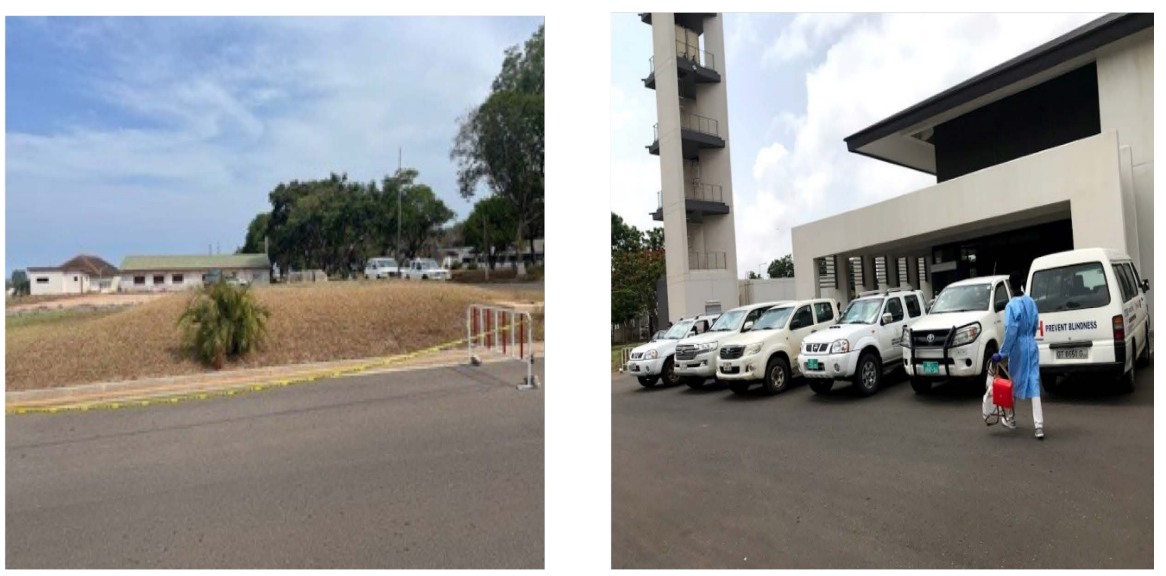

(d)

Figure 1. Sample receipt at the forecourt of the ARL (a). DCOs offloading samples for and completing necessary requirements for submission (b). DCOs submitting samples at night (c). A landmark at the forecourt at the ARL which was cordoned for samples delivered by drones (d). A member of staff retrieves samples delivered by the drone and heads for the sample receipt station.

entrance hall of the ARL, where e-registered samples are verified. The receiving staff at this end verify the completeness of the online registration for samples being investigated for SARS-CoV-2. Samples that do not conform to receipt requirements are returned to the investigators to complete documentation and/or 
sample re-taking. It is worth noting that some hard-to-reach districts and difficult-to-access health facilities in the country submitted suspected COVID-19 samples to NMIMR using drones. Provision was made infront of the ARL, on a cordoned wide stretch of lawn for the drop of packaged samples from these drones and pick up by staff of NMIMR (Figure 1(c) and Figure 1(d)). The drone services were provided by an international company called Zipline International and was supported by the Government of Ghana.

The immediate surroundings and the forecourt of the ARL had enough parking space for "arranged packing" of all the vans and pick-ups brought in by clients and the DCOs for sample submission (Figure 2(a) and Figure 2(b)). Vehicular parking in the spaces assigned was manned and directed by the NMIMR security staff and are "arranged" in first-come first-serve basis.

The samples and accompanying $\mathrm{CI}$ forms are then transported to the sample receipt laboratory at the Virology Department. Here, Research Assistants (RAs) begin sample processing by first assigning unique laboratory IDs, aliquoting into appropriate cryogenic tubes, pooling (in some cases) and nucleic acid extraction. At the peak of the testing, there were two designated sample receipt laboratories, while all the other laboratories in the Virology Department except the cell culture laboratories, were used for the remaining stages of sample processing (sample

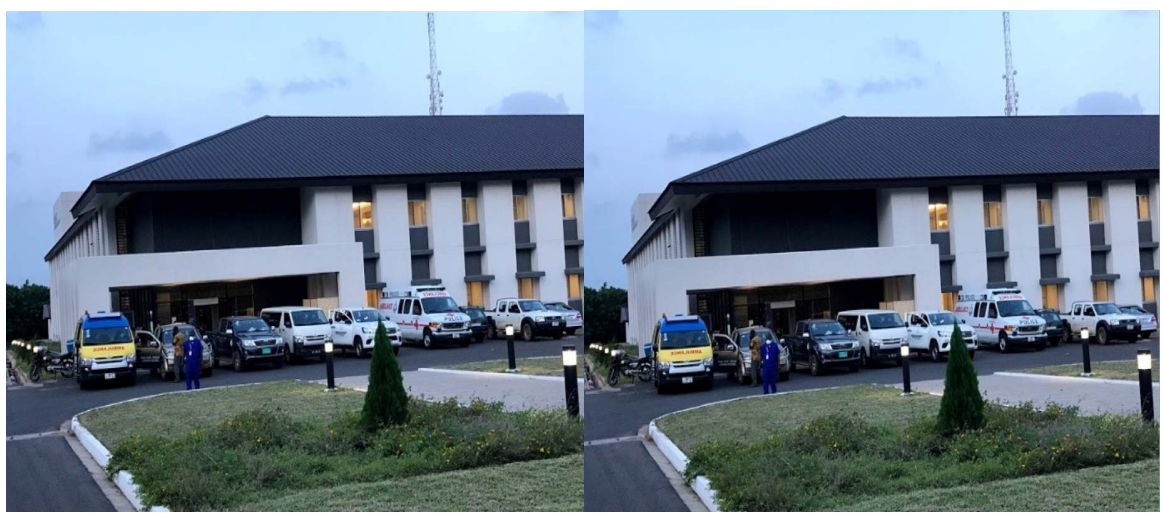

(a)

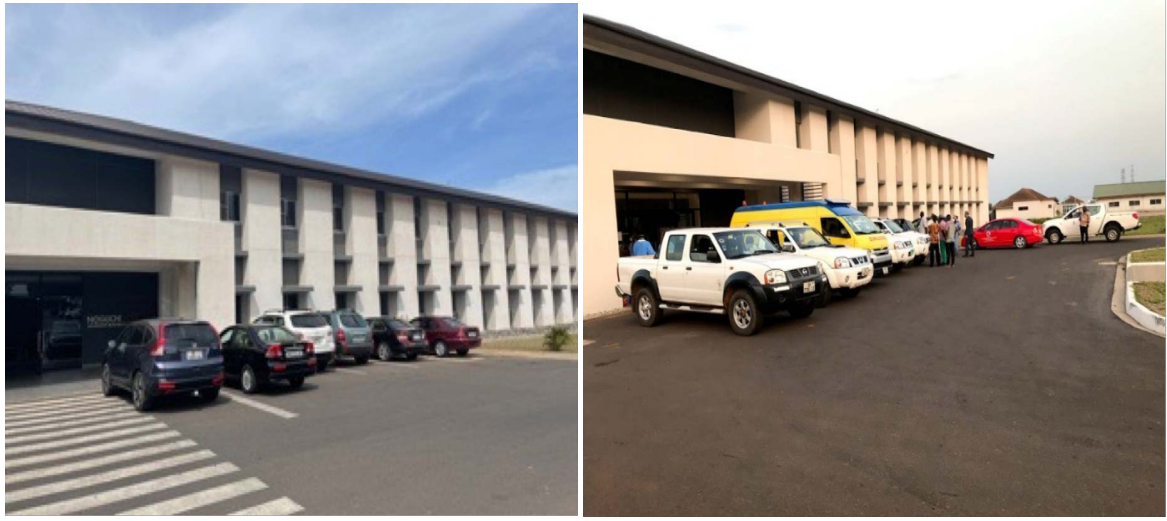

(b)

Figure 2. A photo of the forecourt of the ARL showing (a). A host of vehicles lined up to submit samples (b). Sufficient vehicle parking space. 
aliquoting, sample pooling and RNA extraction). Laboratories in the Bacteriology and Immunology Departments were also used as sample receipt, aliquoting and pooling stations. The sample receipt laboratories had data management staff that used Microsoft Excel spreadsheet for entries of all the information on samples, indicating assigned laboratory IDs and completing all fields as indicated on the case investigation forms. Each laboratory data officer at the end of the day submits the entries to the central database office for merging and reporting.

All the laboratories, including the BSL-3 laboratories, had two biosafety cabinets. One of the cabinets remained for sample aliquoting and pooling (dirty work) while the other cabinet was reserved for RNA extraction and purification and related processes (clean work).

After extraction, the purified RNA extracts are sent back to the main receipt laboratories for data reconciliation before forwarding to the polymerase chain reaction (PCR) suite (Figure 3(a) and Figure 3(b)) for the last stage of the testing process. All the PCR systems in the suite (10 in number) get engaged at one time or the other during the 24 -hour routine testing.

The ARL has a furnished PCR suite, which comprise a Master Mix room, two template addition rooms, [one for ribonucleic acid (RNA) and de-oxyribonucleic acid (DNA) handling], an instrument room that accommodates about nine real-time thermocyclers and eight thermal cyclers for conventional PCR and a furnished post-PCR room for analysing PCR products and a next-generation sequencing platform. The number of real time PCR systems which were five in number at the early stages of testing was doubled through the benevolence of corporate organizations in the course of time. This increased the Institute's capacity to test more samples and reduce turn-around time. In the RNA template addition room, a separate biosafety cabinet (clean bench) is set up for handling and adding positive controls to avoid contamination. The instrument room is equipped with a large refrigerator and biomedical freezer that are used to preserve RNA and DNA pending PCR runs. Completed PCR data are transmitted

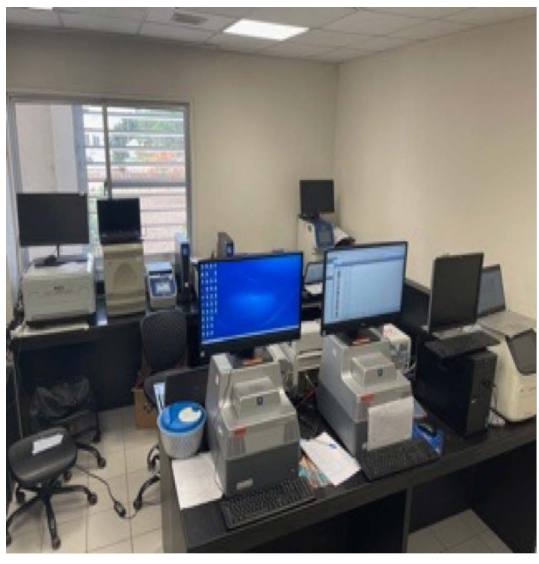

(a)

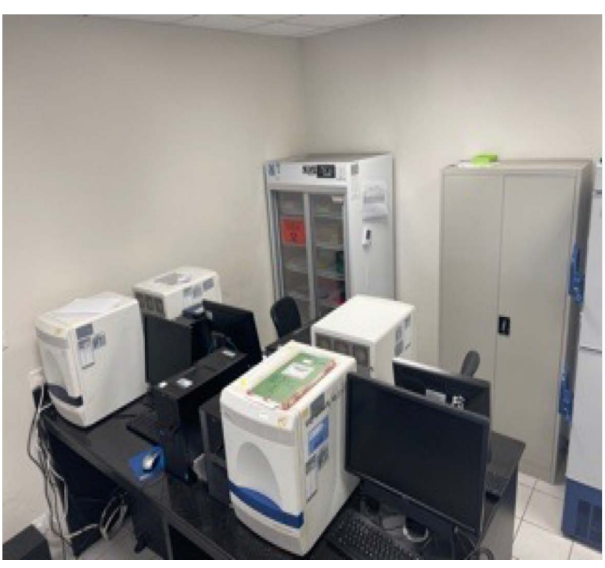

(b)

Figure 3. Shows the 10 real time PCR machines that were used for SARS-CoV-2 testing at the ARL at NMIMR. 
from the instrument room to the data managers via email.

The uni-directional flow approach with regards to movement during processing from master mix preparation through data transmission allowed maximum work efficiency, making it possible to process over four thousand samples daily. The workflow and the expertise of the staff involved reduced the possibility of contamination during routine processing as well as offered maximum protection for staff who worked on SARS-CoV-2 testing and others who meet them. The sheer numbers of suspected samples being submitted daily for testing were overwhelming, so were the CI forms. The compilation and storage of these forms posed a huge challenge till wardrobes were constructed at convenient spaces in the ARL for them (Figure 4(a) and Figure 4(b)).

\subsection{Waste Handling and Disinfection}

The testing processes like every other laboratory procedure generated large volumes of hazardous biological waste. In line with international standards [12], biological waste is decontaminated using a steam process also known as autoclaving before disposal by incineration. The BSL-3 laboratories have two giant autoclaving units which were put into maximum effect in this respect (Figure 5). The two double-door pass-through autoclaves, each with a chamber volume of 1580 litres were used to decontaminate the large volumes of waste that were generated from the laboratory each day. The decontaminated waste is transferred out of the laboratory daily to a designated place where trained transport staff move them to the incinerator. Liquid waste is treated by adding hypochlorite solution and leaving overnight before discarding down the drain with copious amount of water. There were smaller autoclave units in the laboratories that were used for clean sterilization purposes.

The ARL has a coordinated water system. The facility has a central water supply unit, measuring about 80,000 litres that supplies water to all the laboratories in the facility. Each laboratory has two washing sinks, for handwashing and general laboratory use. The consistent flow of water encouraged handwashing

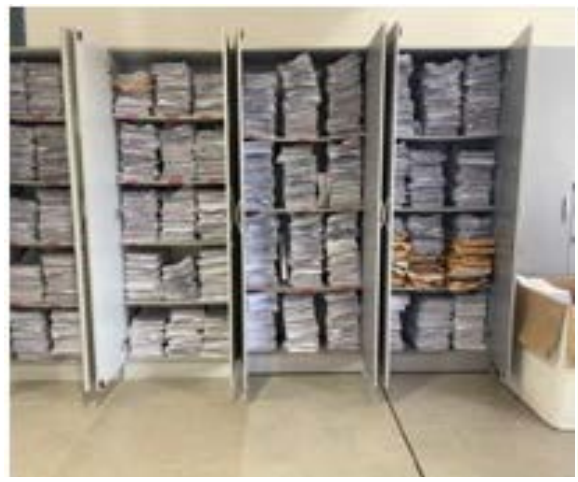

(a)

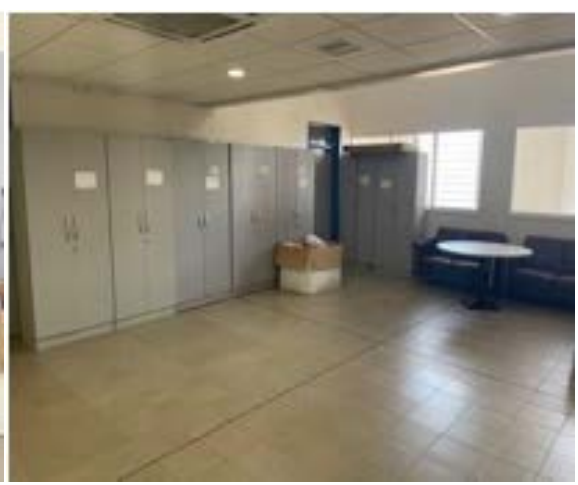

(b)

Figure 4. Shows the storage of CI forms that accompanied samples that were submitted for testing. 

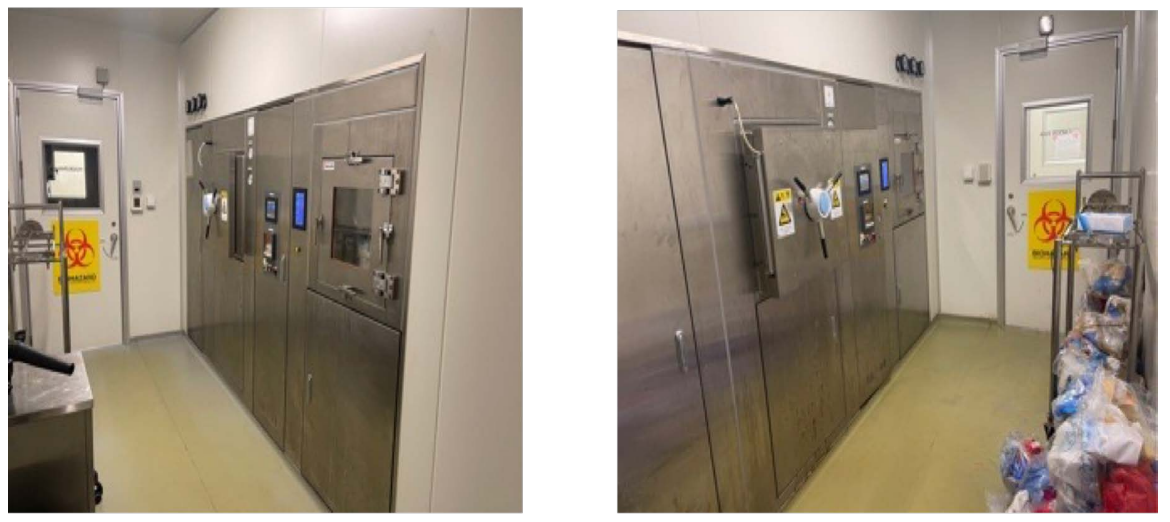

Figure 5. Shows the giant wall-unit autoclaves that were used for disinfection and autoclaving.

among RAs and staff who were engaged with work in the laboratories. As part of good hygiene, automated hand sanitizer dispensers were placed in the spacious corridor of the ARL, only spaced at 30 walking steps (5 metres) apart. The main entrance of the facility is fitted with a walk-through disinfection equipment booth, which staff used during the peak period of the pandemic. Also, the presence of automated extractors, fitted with HEPA-filters in each laboratory was essential in reducing the risk of infection of staff in the laboratories and unintentional exposure of potentially hazardous agents to the environment during sample handling or processing.

\subsection{Data Handling and Reporting}

One of the offices which was meant for technicians at the Virology Department was repurposed into a central data management and reporting room. Here, patient data entered at the various stations within and outside NMIMR, as well as PCR results are merged to complete the testing process. There are passwordprotected computers that were used to report classified test results to investigators through email, phone calls and/or printed hard copies of results. Every report generated by the data team is saved in a folder labelled with the date of reporting and password encrypted for data security reason. He data is regularly back up on an external drive and a sever. Online requested tests were also updated by the data management staff. The data management office operated a customer service desk that received telephone calls with complaints, results request and other enquiries pertaining COVID-19 testing in the Institute. The data office had a waiting area that could accommodate about five guests at any time. The spacious room accommodated up to four data management personnel and space for three visitors from health facilities, who could walk in to inquire about pending results.

\subsection{Walk-In and Commercial Testing Center}

Commercial SARS-CoV-2 PCR tests have become necessary across the globe since it became a requirement for all travellers into many countries to possess a 
PCR-negative certificate. The first commercial tests were done at NMIMR following increasing demands for travelling and walk-in clients who needed to know their COVID-19 status. The commercial stand was set-up at a large space behind the ARL. Clients who access the facility register and make payments to a finance officer after which their personal details as captured in their passports or traveller's certificates are captured into a database. Their residential address, telephone contact and email addresses are also collected to enable Ghana Health Service staff to contact them in the event they test positive for SARS-COV-2. Once registered, with unique laboratory IDs, these same IDs are assigned to sample collections tubes with packaged sterile swabs and given to the client. The client proceeds to designated makeshift cubicles on-site where the respiratory sample is taken. The samples are then transported to the laboratory and processed together with the routine hospital samples. Clients access their test reports at a separate desk at the walk-in centre at the institute or via an internet-based platform, through their email addresses.

\section{Concluding Commentary and Outlooks}

It is obvious that the general success of SARS-CoV-2 testing in Ghana owes to the investment of resources into facilities such as the ARL at the NMIMR, with state-of-the-art equipment for biomedical research. In addition to surveillance testing, the Illumina sequencing platform at ARL was important in the partnership with WACCBIP to publish the first genome data of circulating variants of SARS-CoV-2 in Ghana [6].

The ARL and the expertise of research faculty at the NMIMR tremendously contributed to the take-off and sustenance of SARS-CoV-2 testing in Ghana. Currently, the SARS-CoV-2 laboratory network in Ghana has expanded, comprising twenty-five individual public and private laboratories, with about $30 \%$ of them having the requisite permit to provide certified reports for international travellers after testing in their facilities. It is noteworthy that, many of the laboratories that form a part of the SARS-CoV-2 testing network in Ghana were trained by the staff of NMIMR and have adopted the workflow and protocols developed by and implemented at the NMIMR ARL. These protocols and workflows have been shown to be effective and bio-safe to meet international standards.

In addition, the ARL currently serves as the central laboratory of SARS-CoV-2 testing in Ghana, accommodating the Office of the Coordinator of the National COVID-19 testing laboratories network. The large corridors, stores and cold rooms at the ARL have strategically provided space for the storage of laboratory consumables, supplies and reagent stocks of all national COVID-19 PCR testing laboratories in the country.

The numerous advantages of the ARL clearly spell out a blueprint for testing and diagnosis of infectious diseases which can be modelled in many biomedical and research laboratories. The facility also remains a perfect model for the de- 
velopment of infectious disease centres in Ghana and by extension replicated in other developing countries. The facility which has proven to be excellent, was timely for the country and came in handy to save the blushes of our hitherto fragile health, research, and diagnostic systems. It will therefore be imperative for African governments to establish strategic collaborations to consciously build many more Advanced Research Laboratories to increase testing capacity and biomedical science research. If this is successfully done, the burden of routine diagnosis of infectious diseases during disease outbreaks and pandemics such as the current one will be taken off from Academic Research Centres so they can concentrate on meaningful research that will contribute greatly to scientific technological advancement in medicine, health, and industry.

The gift of the ARL is therefore a timely and priceless gift of friendship from the people and government of Japan for which the government and people of Ghana are extremely grateful.

\section{Acknowledgements}

The authors acknowledge the COVID-19 testing teams at the NMIMR and all other persons who contributed in diverse ways to the successful testing of SARSCOV-2 at the Noguchi Memorial Institute for Medical Research at the University of Ghana from the beginning of the pandemic till date.

\section{Funding Statement}

The authors did not receive any specific funding for this work.

\section{Conflict Statement}

The authors declare no potential conflict of interest with respect to the authorship, preparation and publication of this manuscript. The manuscript was drafted and submitted without any funding nor interference from the Government of Japan nor her representatives in Ghana.

\section{References}

[1] Esbin, M.N., Whitney, O.N., Chong, S., Maurer, A., Darzacq, X. and Tjian, R. (2020) Overcoming the Bottleneck to Widespread Testing: A Rapid Review of Nucleic Acid Testing Approaches for COVID-19 Detection. RNA, 26, 771-783. https://doi.org/10.1261/rna.076232.120

[2] Mohamadian, M., Chiti, H., Shoghli, A., Biglari, S., Parsamanesh, N. and Esmaeilzadeh, A. (2021) COVID-19: Virology, Biology and Novel Laboratory Diagnosis. The Journal of Gene Medicine, 23, e3303. https://doi.org/10.1002/jgm.3303

[3] Lai, C.K.C. and Lam, W. (2021) Laboratory Testing for the Diagnosis of COVID-19. Biochemical and Biophysical Research Communications, 538, 226-230. https://doi.org/10.1016/j.bbrc.2020.10.069

[4] Vandenberg, O., Martiny, D., Rochas, O., van Belkum, A. and Kozlakidis, Z. (2021) Considerations for Diagnostic COVID-19 Tests. Nature Reviews Microbiology, 19, 171-183. https://doi.org/10.1038/s41579-020-00461-Z 
[5] Bonful, H.A., Addo-Lartey, A., Aheto, J.M.K., Ganle, J.K., Sarfo, B. and Aryeetey, R. (2020) Limiting Spread of COVID-19 in Ghana: Compliance Audit of Selected Transportation Stations in the Greater Accra Region of Ghana. PLOS ONE, 15, e0238971. https://doi.org/10.1371/journal.pone.0238971

[6] Ngoi, J.M., Quashie, P.K., Morang'a, C.M., Bonney, J.H., Amuzu, D.S., Kumordjie, S., et al. (2020) Genomic Analysis of SARS-CoV-2 Reveals Local Viral Evolution in Ghana. Experimental Biology and Medicine, 246, 960-970.

[7] Asante, I.A., Adusei-Poku, M., Bonney, H.K., Bonney, E.Y., Odoom, J.K., Obodai, E., et al. (2020) Molecular Diagnosis for the Novel Coronavirus SARS-CoV-2: Lessons Learnt from the Ghana Experience. Ghana Medical Journal, 54, 77-85. https://doi.org/10.4314/gmj.v54i4s.12

[8] Sylverken, A.A., El-Duah, P., Owusu, M., Schneider, J., Yeboah, R., Ayisi-Boateng, N.K., Gorman, R., Adu, E., Kwarteng, A., Frimpong, M., Binger, T., Aryeetey, S., Asamoah, J.A., Amoako, Y.A., Amuasi, J.H., Beheim-Schwarzbach, J., Owusu-Dabo, E., Adu-Sarkodie, Y., Obiri-Danso, K., Corman, V.M., et al. (2021) Transmission of SARS-CoV-2 in Northern Ghana: Insights from Whole-Genome Sequencing. Archives of Virology, 166, 1385-1393. https://doi.org/10.1007/s00705-021-04986-3

[9] Bonney, E.Y., Lamptey, H., Puplampu, P.B. and Kyei, G. (2020) COVID-19 Pandemic: Ten Research Questions Africa Must Answer for Itself. Ghana Medical Journal, 54, 107-12. https://doi.org/10.4314/gmj.v54i4s.17

[10] Corman, V.M., Landt, O., Kaiser, M., Molenkamp, R., Meijer, A., Chu, D.K., et al. (2020) Detection of 2019 Novel Coronavirus (2019-nCoV) by Real-Time RT-PCR. Eurosurveillance, 25, pii=2000045. https://doi.org/10.2807/1560-7917.ES.2020.25.3.2000045

[11] Noguchi, H. (1919) Etiology of Yellow Fever: V. Properties of Blood Serum of Yellow Fever Patients in Relation to Leptospira Icteroides. Journal of Experimental Medicine, 30, 9-12. https://doi.org/10.1084/jem.30.1.9

[12] Gertsman, S., Agarwal, A., O’Hearn, K., Webster, R., Tsampalieros, A., Barrowman, N., et al. (2020) Microwave- and Heat-Based Decontamination of N95 Filtering Facepiece Respirators: A Systematic Review. Journal of Hospital Infection, 106, 536553. https://doi.org/10.1016/j.jhin.2020.08.016

\section{Abbreviations and Acronyms}

NMIMR—Noguchi Memorial Institute for Medical Research;

ARL-Advanced Research Laboratories;

DCO_Disease Control Officer;

GHS-Ghana Health Service;

WHO-World Health Organization;

NIC-National Influenza Centre;

PCR-Polymerase Chain Reaction;

JICA-Japan International Cooperation Agency;

CI-Case Investigation. 